\title{
Analysis of cutaneous pigmentation in a sample
}

\author{
Juan Pablo Castanedo-Cazares', Diana Hernández-Blanco¹, Juan Diego García-Cortés², \\ Liliana Medina-Aguilar ${ }^{1}$ and Bertha Torres-Álvarez ${ }^{1}$ \\ ${ }^{1}$ Dermatology Department, Hospital Central Dr. Ignacio Morones Prieto; '2Department of Immunology and Molecular Biology, Faculty of Chemical \\ Sciences, Universidad Autónoma de San Luis Potosí, San Luis Potosí. S.L.P., Mexico
}

\begin{abstract}
Background: The blend of hemoglobin, carotenes, and melanin defines the skin color. Constitutive pigmentation is genetically determined, facultative color is induced when skin is exposed to environment. The objective was to quantify both pigmentations in a sample of Mexican population and to analyze its relationship with sex, age, and phototype. Methods: We evaluated 259 individuals during the winter. Skin colorimetry was obtained by diffuse reflectance spectrometry, using the International Commission of Illumination coordenates. $L^{*}{ }^{*}{ }^{*}{ }^{*}$ parameters were measured and the individual typological angle (ITA) was estimated from forehead, thorax, neck, forearms, and buttocks areas. Results: Facultative pigmentation differed from constitutive in $L^{*}$, $a^{*}$, and ITA values. In men, $L^{*}$ and ITA parameters were lower. Constitutive pigmentation was similar between sexes. Phototypes III, IV, and $V$ showed differences in $L^{*}, b^{*}$, and ITA ${ }^{\circ}$. Facultative values such as $L^{*}, a^{*}$, ITA ${ }^{\circ}$, and the constitutive $a^{*}$ reduce as age increases. Conclusions: The cutaneous tones of a sample of population were quantified recognizing their values for white, light brown, and dark brown skin. A reference frame for research related to cutaneous pigmentation in Mexico is presented.
\end{abstract}

KEY WORDS: Spectrophotometry. Colorimetry. Pigmentation. Phototype.

\section{Introduction}

Human skin is diverse in color, since it exhibits a range of white, brown and black tones. This characteristic is used to distinguish or classify different ethnicities ${ }^{1}$. Its appearance is due to the blend of pigments or chromophores that absorb particular electromagnetic wavelengths and transmit or reflect them to the visible light spectrum ${ }^{2-4}$. The most important are oxyhemoglobin, which reflects the red color, deoxyhemoglobin, which reflects blue, carotenes, which reflect yellow-orange and melanin, which reflects the brown color ${ }^{3,5,6}$. Melanin is the main pigment that determines dark or light tone according to its concentration in the epidermis $^{2,3}$. This substance's biological function is to protect cells from ultraviolet (UV) radiation and its synthesis is increased in response to environmental stimuli ${ }^{7-9}$.

The genetically-determined color that is observed in areas of the body that are isolated from the environment and/or protected by clothing represents the constitutive pigmentation ${ }^{10}$. Darkening caused by UV exposure corresponds to facultative pigmentation. This tanning is evident in exposed areas such as the face, neck, arms and forearms, and it gradually diminished when the stimulus ceases ${ }^{8,10}$. Therefore, observation of cutaneous pigmentation and its changes suggests tolerance or predisposition for the development of solar radiation-associated pathologies ${ }^{9,11}$. If UV aggression exceeds the protection or adjustment mechanisms,

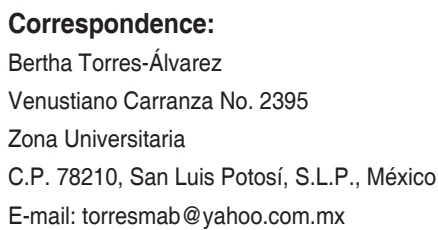

Gac Med Mex. 2018;154:55-59

Contents available at PubMed www.gacetamedicademexico.com 
burns, dyschromia, aging and benign or malignant neoplasms will appear on the most exposed areas of the $\mathrm{skin}^{8,12,13}$.

Owing to the subjective perception of skin color, Fitzpatrick's classification is used in medicine to recognize the type of skin; this classification evaluates its biological response to sun exposure distinguishing six skin tones or phototypes, with 3 variants of white skin, 2 brown and one black ${ }^{14}$. However, skin color objective quantification requires tridimensional localization of the red, green and blue colors ${ }^{3}$. These coordinates are based on the absorption bands of the 3 pigments present in human retina cones ${ }^{15}$. Colorimetry consists in objectively measuring these three values, as well as their luminescence, by means of some method such as the one proposed by the International Commission on Illumination (e.g., $\left.L^{*} a^{*} b^{*}\right)^{3,15}$. This process enables a numerical representation of any color present in nature ${ }^{3,4,15}$. The purpose of this work was to quantify constitutive and facultative pigmentation in Mexican adult subjects using a diffuse reflectance spectrometer and to analyze their relationship with gender, age and phototype.

\section{Methods}

By means of informed consent and local ethics committee approval (3611), voluntary subjects older than 18 years were invited to participate during the winter period from November to February. By means of non-probabilistic sampling, users and visitors of the Hospital Central "Dr. Ignacio Morones Prieto" of the city of San Luis Potosí were included. Subjects with any skin tone who had the Mexican nationality since at least 3 previous generations were selected. Subjects with skin and systemic conditions, consumption of medications, pregnancy or breastfeeding within the previous 6 months were excluded.

The type of skin was assessed according to Fitzpatrick's classification ${ }^{14}$, which examines the cutaneous response of an individual after 1 hour of solar exposure at midday in summer. This interval contains approximately $90 \mathrm{mJcm}^{2}$ of UV radiation in most part of the Mexican Republic ${ }^{9}$. After contact, and according to the existence of skin burn and/or pigmentation at 24 hours and 7 days, respectively, the skin responses are grouped as: I, always burns, never tans; II, usually burns and hardly tans; III, burns with minimal tanning; IV, occasionally burns, tans well; V, very rarely burns, tans very easily and $\mathrm{VI}$, never burns, permanent dark skin ${ }^{9,14}$.
Skin colorimetry was measured with a diffuse reflectance colorimeter (Chromameter 310, Minolta, Osaka, Japan), using the International Commission of Illumination color spaces. They consist of a luminosity central axis $L^{*}$ with values from black (0) to white (100) and two orthogonal axes that represent chromaticity: $a^{*}$ describes colors from red $(+50)$ to green $(-50)$, and $b^{*}$, colors from blue $(-50)$ to yellow $(+50)^{4}$.

Facultative pigmentation was estimated by calculating the averages of the measurements obtained at 5 body sites: supra-glabellar region in the forehead, upper chest supraclavicular zone, posterior neck at the level of the sixth cervical vertebra and lateromedial portion of both forearms above the epicondyle. Constitutive pigmentation was obtained from the average of measurements on the upper external and lower internal quadrants of the gluteal area. The instrument was calibrated prior to each measurement and the analyzed value was the mean of 3 consecutive readings on each one of the described areas.

Both pigmentation values were classified according to $\mathrm{ITA}^{\circ}$. This value corresponds to central luminescence bi-dimensional projection angular segmentation values $\left(L^{*}=50\right)$ versus yellow chromaticity initial value $\left(b^{*}=0\right)^{6}$. It is obtained with the formula: ITA $=[$ Arc$\operatorname{Tan}\left(L^{*}-50 / b^{*}\right] \times(180 / 3.14159)$. This parameter enables skin classification in 6 groups (very light $>55>$ light $>41$ intermediate $>28>\tan >10$ brown $>-30>$ dark $)^{6,16}$.

Approximately $95 \%$ of the Mexican population phototypes include III, IV or V9,17. Therefore, the sample size was calculated to be of at least 16 subjects per group according to the following considerations: at least $5 \%$ difference in gluteal area luminosity $\left(\mathrm{L}^{\star}\right)$ between phototypes II and V (e.g. 61 for type III and 58 for V). Intermediate distribution of means, standard deviation of 1.8 and $95 \%$ confidence interval, twotailed, alpha value of 0.05 and beta of 0.9 were used. The descriptive analysis was performed using central tendency measures. Data comparison was carried out with Student's t-test, analysis of variance and correlation tests. Everything at a level of confidence of $95 \%$ and a level of significance of $5 \%$, using the JMP program, version 8.0 (Cary, NC, USA).

\section{Results}

The characteristics of 259 subjects were analyzed. Average age was $34 \pm 14$ years (range: 18 to 75 ). Sixty-five percent were females $(n=169)$ and $35 \%$ $(n=90)$ were males. Phototypes in the sample were 
II ( $\mathrm{n}=17)$, III $(\mathrm{n}=49)$, IV $(\mathrm{n}=82)$ and V $(\mathrm{n}=111)$. There were no differences in average age or in gender proportions between the four groups.

$\mathrm{L}^{*}, \mathrm{a}^{*}, \mathrm{~b}^{*}$ and facultative pigmentation ITA $\mathrm{A}^{\circ}$ average values were $57.3 \pm 4.7,10.2 \pm 2.4,15.8 \pm 2.5$ and 24.2 \pm 14.6 , respectively. As regards constitutive pigmentation, $\mathrm{L}^{*}, \mathrm{a}^{*}, \mathrm{~b}^{*}$ and $\mathrm{ITA}^{\circ}$ mean values were $62.7 \pm 4.5$, $7.7 \pm 2.9,14.6 \pm 3$ and $40.3 \pm 15.1$, respectively. All these parameters, except for $b^{*}$, showed significant differences between both pigmentations $(p<0.01)$.

Between-gender comparison showed significant differences in facultative pigmentation $L^{*}$ and ITA ${ }^{\circ}$ values, since these were higher in males. However, red and yellow color quantification was similar for both groups in both pigmentations. These data are shown in table 1.

$\mathrm{ITA}^{\circ}$ calculation for constitutive pigmentation established that $15.4 \%(n=40)$ were very light, $38.9 \%$ $(n=101)$ were light, $27.4 \%(n=71)$ were intermediate, $13.2 \%(n=34)$ were tanned, $5.1 \%(n=13)$ were brown and $0 \%$ dark. Facultative pigmentation ITA ${ }^{\circ}$ showed a proportion of $0.7 \%(n=2)$ for very light skin, $10.4 \%(n=27)$ for light, $32 \%(n=83)$ for intermediate, $38.9 \%(n=101)$ for tanned, $17.7 \%(n=46)$ for brown and $0 \%$ dark. This distribution is shown in figure 1 .

The facultative and constitutive pigmentation analysis with regard to Fitzpatrick phototypes demonstrated significant differences for both pigmentations in the $L^{*}, b^{*}$ and ITA ${ }^{\circ}$ values (ANOVA, $p<0.001$ ). Facultative pigmentation showed increases in the $L^{*}$ value of $7.5 \%, 7.5 \%, 9.2 \%$ and $8.5 \%$ for phototypes II, III, IV and $\mathrm{V}$, respectively, with regard to constitutive pigmentation. There were no changes in red color $\left(a^{*}\right)$ between phototypes. These data are summarized in table 2.

As regards the association between pigmentation and age, the facultative variant $L^{*}$ and ITA ${ }^{\circ}$ values were observed to decrease with increasing age (R: -0.17 and -0.15 , respectively; $p<0.01$ ). The same trend was observed with the red tone $\left(a^{*}\right)$ representation $(R:-0.15$; $p=0.01$ ). Constitutive pigmentation showed an inverse relationship between red color intensity and age increase $(R:-0.4 ; p<0.001)$. No changes were determined in the $L^{*}, b^{*}$ and ITA $A^{\circ}$ values with regard to age. These findings are depicted in figure 1.

\section{Discussion}

Pigmentation is a physical trait that favors the distinction between different human groups. Its quantification has anthropological, social, psychological and
Table 1. Comparison of $L^{*}, a^{*}, b^{*}$ and ITA ${ }^{\circ}$ values between males and females for environment-exposed and non-exposed areas in 259 subjects

\begin{tabular}{|c|c|c|c|c|c|c|}
\hline \multicolumn{4}{|c|}{ Facultative } & \multicolumn{3}{|c|}{ Constitutive } \\
\hline & Male & Female & $P$ & Male & Female & $P$ \\
\hline$L^{*}$ & $58 \pm 3.9$ & $56.1 \pm 5.7$ & 0.001 & $63.1 \pm 4.4$ & $61.8 \pm 5.4$ & 0.06 \\
\hline$a^{*}$ & $9.7 \pm 2.2$ & $10.2 \pm 2.6$ & 0.08 & $7.1 \pm 2.7$ & $7.2 \pm 2 . \overline{9}$ & 0.09 \\
\hline$b^{*}$ & $15.8 \pm 2.4$ & $15.7 \pm 2.6$ & 0.8 & $14.3 \pm 2.8$ & $15 \pm 3.2$ & 0.24 \\
\hline $\mathrm{ITA}^{\circ}$ & $26.5 \pm 12.2$ & $20 \pm 17.5$ & 0.006 & $41.9 \pm 14$ & $37.4 \pm 16.8$ & 0.08 \\
\hline
\end{tabular}

Data show $L^{*}\left(\right.$ luminosity) $a^{*}\left(\right.$ red) $b^{*}$ (blue) and ITA (individual typology angle) mean \pm standard deviation. Facultative corresponds to skin exposed to solar radiation; constitutive to non-exposed skin. $P<0.05$, Student's t-test.

healthcare-related implications ${ }^{10,13,18}$. This variable represents an adaptive process to the geographic surroundings with clinical repercussions. Therefore, measuring pigmentation in a population is relevant, since it translates its predisposition for suffering environmental harm, as well as the possible presence of local or systemic pathologies $5,16,19$. This work represents the first study in Mexico that measures skin coloration by means of diffuse reflectometry. The study was carried out in winter in order to minimize the influence of the intense solar exposure experienced by the population of our country for most part of the year ${ }^{17,20}$.

Our study found a significant increase in values that indicate the presence of melanin and blood contents in the facultative pigmentation in both genders. However, males showed higher values than females. Melanin increased synthesis in this group is consistent with reports referring longer time of solar exposure during occupational and recreational activities ${ }^{17}$. As regards constitutive pigmentation, no differences were found between both genders. This could mean that genetic sensitivity to UV radiation in our population is not influenced in a determinant form by this variable, as reported in other studies ${ }^{9,15,16,19}$.

With regard to Fitzpatrick clinical classification, we identified 4 of the 6 described types. Although reflectometry did not distinguish differences between light phototype values such as II and III, the $L^{*}$ and ITA parameters identified 3 skin tones or categories, both in facultative and constitutive pigmentation. These would correspond to $\mathrm{V}$ (dark brown), IV (moderate brown), and to groups II-III (fair). Unlike dark brown and fair groups, the divergence between both pigmentations was more accentuated in the moderate brown group. This higher contrast might represent a larger adaptive effort of this group with regard to the higher 

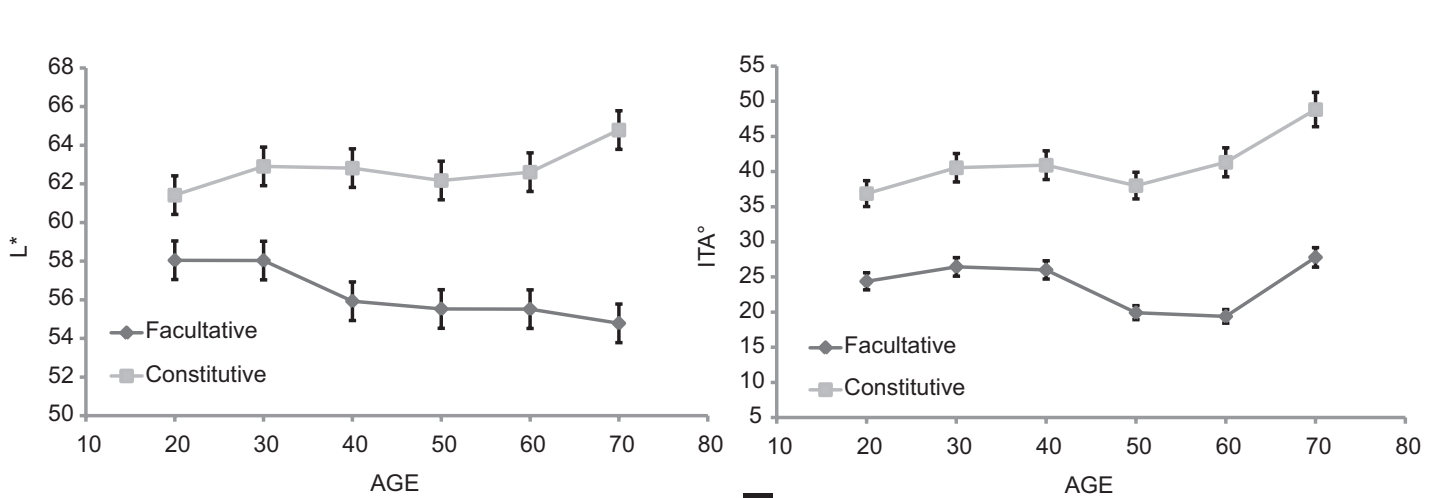

A

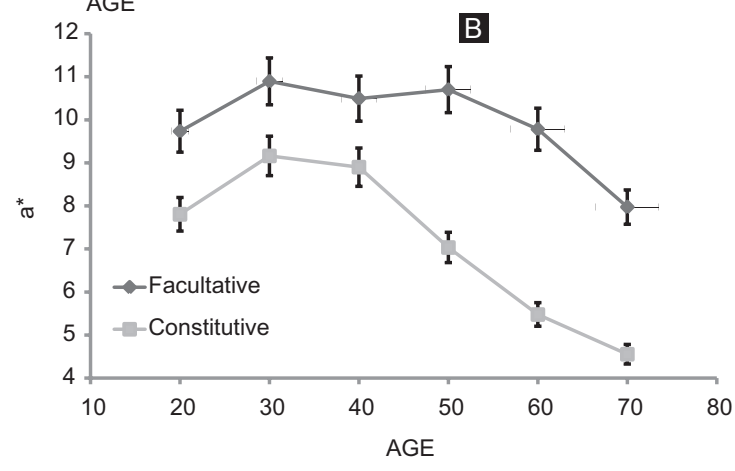

Figure 1. $L^{*}$, ITA (Individual Typology Angle) and $a^{*}$ values variation with regard to chronologic age of assessed individuals. (A) Luminosity is reduced as age advances in facultative pigmentation $(R-0.17, P<0.01)$, in contrast with constitutive pigmentation, which remains unchanged. (B) $I T A^{\circ}$ is reduced as age increases in facultative pigmentation $(R-0.15, P=0.01)$, and is not modified in constitutive pigmentation. (C) $a^{*}$ value decreases as age increases in facultative $(R-0.15, P=0.01)$, as well as in constitutive pigmentation $(R-0.39, P<0.001)$. The graph shows values grouped by decade (mean $\pm S D$ ).

Table 2. Differences between the $L^{*}, a^{*}, b^{*}$ and ITA ${ }^{\circ}$ values according to the different phototypes in exposed and non-exposed skin areas in 259 subjects

\begin{tabular}{|c|c|c|c|c|c|}
\hline & \multicolumn{4}{|c|}{ Facultative } & \multirow{2}{*}{$\frac{\rho}{P}$} \\
\hline & II & III & IV & v & \\
\hline$L^{*}$ & $62.17 \pm 3.12$ & $61.19 \pm 3.87$ & $57.82 \pm 4.14^{\mathrm{a}}$ & $54.66 \pm 3.74^{b}$ & $<0.001$ \\
\hline$a^{*}$ & $10.4 \pm 1.78$ & $9.76 \pm 2.25$ & $9.9 \pm 2.61$ & $10.2 \pm 1.44$ & 0.2 \\
\hline$b^{*}$ & $15.18 \pm 2.53$ & $14.29 \pm 2.5$ & $15.22 \pm 1.99$ & $17.09 \pm 2.28^{b}$ & $<0.001$ \\
\hline \multirow[t]{3}{*}{$\mathrm{ITA}^{\circ}$} & $38.47 \pm 9.85$ & $37.18 \pm 8.04$ & $26.39 \pm 12.71^{a}$ & $14.78 \pm 11.84^{b}$ & $<0.001$ \\
\hline & \multicolumn{4}{|c|}{ Constitutive } & $\frac{5}{9}$ \\
\hline & II & III & IV & v & $P$ \\
\hline$L^{*}$ & $67.25 \pm 3.14$ & $66.17 \pm 3.12$ & $63.79 \pm 3.46^{a}$ & $59.7 \pm 4.7^{b}$ & $<0.001$ \\
\hline$a^{*}$ & $8.49 \pm 2.77$ & $7.13 \pm 2.85$ & $7.18 \pm 2.97$ & $7.85 \pm 2.5$ & 0.06 \\
\hline$b^{*}$ & $13.03 \pm 1.87$ & $12.09 \pm 2.34$ & $13.88 \pm 2.46^{a}$ & $16.51 \pm 2.61^{b}$ & $<0.001$ \\
\hline $\mathrm{ITA}^{\circ}$ & $52.44 \pm 8.15$ & $53 \pm 6.69$ & $44.49 \pm 10.84^{a}$ & $29.88^{\prime} \pm 14.38^{b}$ & $<0.001$ \\
\hline
\end{tabular}

Data show $\mathrm{L}^{*}$ (luminosity), $\mathrm{a}^{*}$ (red), $\mathrm{b}^{*}$ (blue) and ITA (Individual Typology Angle) mean \pm standard deviation. Facultative corresponds to skin exposed to solar radiation; constitutive, to

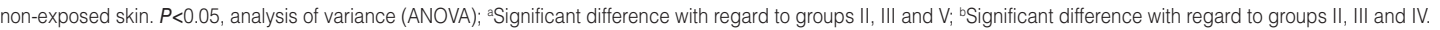

UV tolerance of the dark brown population ${ }^{9,16}$. The poor contrast between both pigmentations in the fair skin group demonstrates its limited capability to respond to environmental aggression ${ }^{8,10,14}$.

Another finding was facultative pigmentation increase as age advances. This may be related to melanogenesis increase as an intrinsic protection mechanism due to structural cutaneous weakening and/or to a reduced melanic clearance capability inherent to skin aging ${ }^{8,13,21}$. However, constitutive pigmentation was similar between the different age groups that were analyzed. On the other hand, a reduction in the 
red tone was observed as age increases in both pigmentations, although it was found to be more pronounced in the constitutive type. This color indicates the presence of blood, and it is probable that our findings are due to the reduced cutaneous vascularity that accompanies chronologic aging ${ }^{8,22}$.

Identifying the values of the three chromatic axes that make up the diverse skin tonalities is relevant for medicine in our country, since it favors grouping or typing of our population. This enables estimating the probability of suffering from pigmentation disorders, photoaging, as well as benign or malignant tumors ${ }^{9,13}$. It also facilitates for preventive measures to be established since early stages of life in the fair skin population ${ }^{9,12}$. On the other hand, identification of extreme chromatic values suggests the presence of pathologies that modify integumentary color or can be useful to monitor them. Some examples are melanogenesis and angiogenesis disorders, as well as deposits of endogenous pigments, such as bilirubin, or exogenous, such as heavy metals ${ }^{5}$.

In conclusion, we obtained the skin tones qualitative ranges in a sample of Mexican population using the $\mathrm{L}^{*} \mathrm{a}^{*} \mathrm{~b}^{*}$ coordinates and the ITA $\mathrm{A}^{\circ}$. A blend of tones was observed that are distinguished as fair, moderate brown and dark brown skin. Constitutive pigmentation was similar between genders and different age groups. Facultative pigmentation increases as age advances and is more profuse in the male population. These values represent a frame of reference for future studies.

\section{References}

1. Relethford JH. Race and global patterns of phenotypic variation. Am J Phys Anthropol. 2009;139:16-22.

2. Anderson RR, Parrish JA. The optics of human skin. J Invest Dermatol. 1981;77:13-9.

3. Takiwaki $\mathrm{H}$. Measurement of skin color: practical application and theoretical considerations. J Med Invest. 1998;44:121-6.

4. Weatherall IL, Coombs BD. Skin color measurements in terms of CIELAB color space values. J Invest Dermatol. 1992;99:468-73.

5. Ortonne JP. Normal and abnormal skin color. Ann Dermatol Venereol. 2012;139 Suppl: S125-9.

6. Chardon A, Cretois I, Hourseau C. Skin colour typology and suntanning pathways. Int J Cosmet Sci. 1991;13:191-208.

7. Nielsen KP, Zhao L, Stamnes JJ, et al. The importance of the depth distribution of melanin in skin for DNA protection and other photobiological processes. J Photochem Photobiol B. 2006;82:194-8.

8. Hönigsmann $\mathrm{H}$. Erythema and pigmentation. Photodermatol Photoimmunol Photomed. 2002;18:75-81.

9. Castanedo Cázares JP, Torres Álvarez B, Sobrevilla Ondarza S, et al. Estimación del tiempo de exposición solar para quemadura en población mexicana. Gac Med Mex. 2012;148:243-7.

10. Quevedo WC, Fitzpatrick TB, Pathak MA, et al. Role of light in human skin color viariation. Am J Phys Anthropol. 1975;43:393-408.

11. Damian DL, Matthews YJ, Phan TA, et al. An action spectrum for ultraviolet radiation-induced immunosuppression in humans. $\mathrm{Br} \mathrm{J}$ Dermatol. 2011;164:657-9.

12. Castanedo-Cázares JP, Martínez-Rosales K, Hernández-Blanco D, et al. In vitro assessment of commercial sunscreens available in Latin America. Invest Clin. 2014;55:142-54.

13. Diffey BL. Ultraviolet radiation and human health. Clin Dermatol. 1998;16:83-9.

14. Fitzpatrick TB. The validity and practicality of sun-reactive skin types I through VI. Arch Dermatol. 1988;124:869-71.

15. Serup J, Agner T. Colorimetric quantification of erythema--a comparison of two colorimeters (Lange Micro Color and Minolta Chroma Meter CR200) with a clinical scoring scheme and laser-Doppler flowmetry. Clin Exp Dermatol. 1990;15:267-72.

16. Del Bino S, Sok J, Bessac E, et al. Relationship between skin response to ultraviolet exposure and skin color type. Pigment Cell Res. 2006;19:606-14.

17. Castanedo-Cázares JP, Torres-Alvarez B, Medellín-Pérez ME, et al. Conocimientos y actitudes de la población mexicana con respecto a la radiación solar. Gac Med Mex. 2006;142:451-5.

18. Jablonski NG, Chaplin G. Human skin pigmentation as an adaptation to UV radiation. Proc Natl Acad Sci U S A. 2010;107 Suppl:8962-8.

19. Yun IS, Lee WJ, Rah DK, et al. Skin color analysis using a spectrophotometer in Asians. Skin Res Technol. 2010;16:311-5.

20. Castanedo-Cázares JP, Torres-Álvarez B, Portales-González B, et al. Análisis de la radiación solar ultravioleta acumulada en México. Rev Med Inst Mex Seguro Soc. 2016;54:26-31.

21. Costin GE, Hearing VJ. Human skin pigmentation: melanocytes modulate skin color in response to stress. FASEB J. 2007;21:976-94.

22. Tsuchida $Y$. The effect of aging and arteriosclerosis on human skin blood flow. J Dermatol Sci. 1993;5:175-81. 